EPJ Web of Conferences 69, 00023 (2014)

DOI: $10.1051 /$ epjconf / 20146900023

(C) Owned by the authors, published by EDP Sciences, 2014

\title{
Comparison between cross sections, saddle point and scission point barriers for the ${ }^{32} \mathrm{~S}+{ }^{24} \mathrm{Mg}$ reaction
}

\author{
T. J. Santos ${ }^{1, a}$ and B. V. Carlson ${ }^{1}$ \\ ${ }^{1}$ Instituto Tecnológico de Aeronáutica
}

\begin{abstract}
One of the principal characteristics of nuclear multifragmentation is the emission of complex fragments of intermediate mass. The statistical multifragmentation model has been used for many years to describe the distribution of these fragments. An extension of the statistical multifragmentation model to include partial widths and lifetimes for emission, interprets the fragmentation process as the near simultaneous limit of a series of sequential binary decays. In this extension, the expression describing intermediate mass fragment emission is almost identical to that of light particle emission. At lower temperatures, similar expressions have been shown to furnish a good description of very light intermediate mass fragment emission. However, this is usually not considered a good approximation to the emission of heavier fragments. These emissions seem to be determined by the characteristics of the system at the saddle-point and its subsequent dynamical evolution rather than by the scission point. Here, we compare the barriers and decay widths of these different formulations of intermediate fragment emission and analyze the extent to which they remain distinguishable at high excitation energy.
\end{abstract}

\section{Introduction}

It is a well-established experimental fact that a nuclear system breaks up into several smaller nuclei (complex fragments), as well as protons and neutrons, when a large amount of energy (on the order of 2 or more $\mathrm{MeV}$ per nucleon) is deposited in it. This process of nuclear multifragmentation is observed in collisions between heavy ions at energies ranging from a few tens to a few hundreds of $\mathrm{MeV}$ per nucleon.

Both theoretical and experimental studies of the phenomenon have received great attention from the nuclear physics community, initially due the fact that the fragment charge distribution follows a power law, which was interpreted as a sign of a phase transition in nuclear matter. However, several subsequent studies have shown that other mechanisms can also explain such behavior.

The interest in studying the process of nuclear multifragmentation is not exhausted by the prospect of observing such a transition phase. In fact, understanding how nuclear matter behaves when heated and compressed, as occurs in the early stages of heavy-ion nuclear reactions leading to nuclear multifragmentation, is also of great importance in astrophysics for understand the evolution of supernovae. Moreover, this phenomenon is of great theoretical interest in nuclear physics. A complete study of this process would require the ability to describe the dynamical evolution of a quantum many-body

\footnotetext{
ae-mail: nztiago@gmail.com
} 
system of strongly interacting constituents. An exact solution of this problem is beyond the the limits of existing computational resources and formal theoretical tools. Due to the complexity of the dynamics of such a many-body system and the richness of phenomena that it presents, a large number of models and approaches have been used to describe its various facets.

The statistical approach to the problem assumes that the collision between two nuclei leads to a hot compound system in thermodynamical equilibrium. Unlike dynamical approaches, statistical models do not attempt to describe the evolution of the system from the initial stages of the collision.

\section{Statistical emission model}

One of the principal characteristics of nuclear multifragmentation is the emission of complex fragments of intermediate mass. The statistical multifragmentation model[1-4] provides a reasonably good description of the distribution of intermediate mass fragments. However, it does not furnish a complete physical description of the statistical decay, as it does not provide an estimate of the decay widths and lifetimes for emission. The model assumes that fragments are formed simultaneously during the final stage of the expansion of the excited nuclear system. When the multifragmentation statistical model is extended to include the calculation of partial widths, it can be interpreted as the nearly simultaneous decay limit of a sequential emission model. [5]

To obtain such a model, one first considers the multifragmentation of an initial nucleus $Z_{0}, A_{0}$ to be only approximately simultaneous. After organizing the fragments into the two fragments that are the first to separate. one can then sum over all partitions that separate into the same first two fragments, $Z_{1}, A_{1}$ and $Z_{2}, A_{2}$. The rate at which this separation occurs is just the rate at which the two fragments separate beyond their radius of nuclear interaction. This can be written as

$$
\begin{aligned}
& -\frac{d}{d t} \omega_{t o t}\left(\varepsilon_{0}, Z_{0}, A_{0}\right)_{\rightarrow Z_{1} A_{1}, Z_{2} A_{2}}=\frac{1}{(2 \pi \hbar)^{3}} \int d^{3} p d^{3} r \frac{\hat{r} \cdot \vec{p}}{\mu} \theta(\hat{r} \cdot \vec{p}) \delta(r-R) \\
& \quad \times \int \omega_{t o t}\left(\varepsilon_{1}, Z_{1}, A_{1}\right) \omega_{t o t}\left(\varepsilon_{2}, Z_{2}, A_{2}\right) d \varepsilon_{1} d \varepsilon_{2} \delta\left(\varepsilon_{0}-Q-V_{B}-\frac{p^{2}}{2 \mu}-\sum_{j=1}^{2} \varepsilon_{j}\right),
\end{aligned}
$$

where $\omega_{\text {tot }}(\varepsilon, Z, A)$ is the total density of states of nucleus $Z, A$ at excitation energy $\varepsilon, Q$ is the Q-value of the reacton and $V_{B}$ is the effective potential barrier. This expression can be reduced to

$$
\begin{aligned}
2 \pi \Gamma_{Z_{0} A_{0} \rightarrow Z_{1} A_{1}, Z_{2} A_{2}} \omega_{t o t}\left(\varepsilon_{0}, Z_{0}, A_{0}\right)=\int d e \frac{2 \mu e}{\pi \hbar^{2}} \sigma_{a b s, Z_{1} A_{1}+Z_{2} A_{2}}(e) \\
\quad \times \int \omega_{t o t}\left(\varepsilon_{1}, Z_{1}, A_{1}\right) \omega_{t o t}\left(\varepsilon_{2}, Z_{2}, A_{2}\right) d \varepsilon_{1} d \varepsilon_{2} \delta\left(\varepsilon_{0}-Q-e-\varepsilon_{1}-\varepsilon_{2}\right),
\end{aligned}
$$

Here the derivative on the left-hand side has been rewritten in terms of the partial decay width, $\Gamma_{Z_{0} A_{0} \rightarrow Z_{1} A_{1}, Z_{2} A_{2}}$ and the absorption cross section has been substituted for the geometrical cross section

$$
\pi R^{\wedge} 2\left(1-V_{B} / e\right) \rightarrow \sigma_{a b s, Z_{1} A_{1}+Z_{2} A_{2}}(e) .
$$

In this decay model, intermediate mass fragment emission is mathematically described by an expression almost identical to the Weisskopf approximation to light particle emission. At lower temperatures, similar expressions have been shown to furnish a good description of very light intermediate mass fragment emission [6] but not of the emission of heavier fragments, which seems to be determined by the transition density at the saddle-point rather than at the scission point. [7-9] Although the saddle point model is essentially identical to the transition state model developed to describe the fission of heavier systems, we do not expect a significant energy difference between the saddle and 

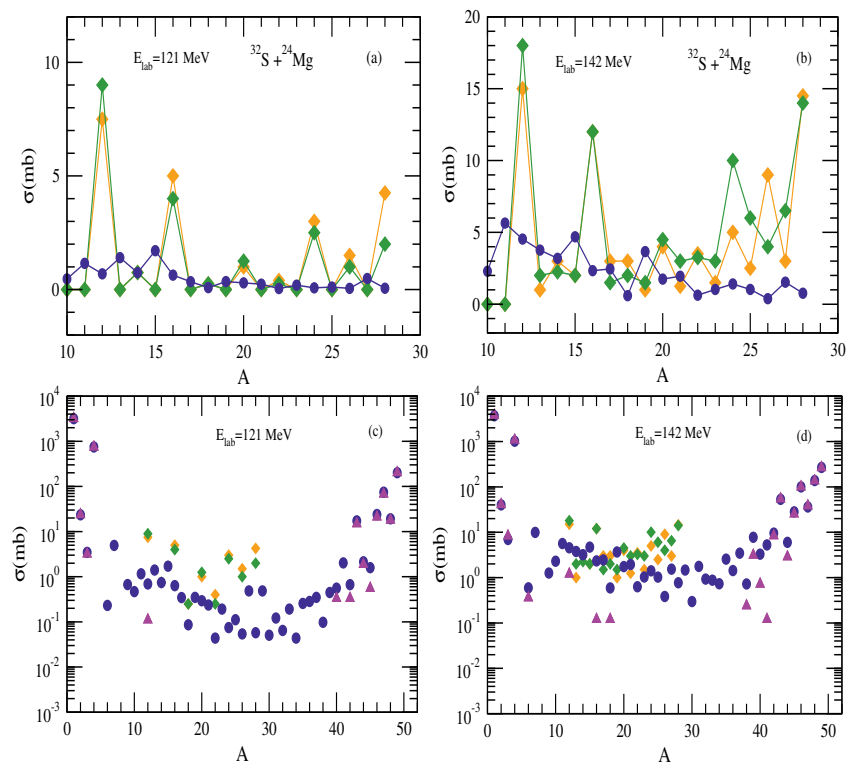

Figure 1. Experimental (green) data and calculated mass distributions, (in yellow, calculated from the critical distance model based on the saddle point [9], in blue, obtained from the Sierk barriers and in pink, calculated using the São Paulo potential). In (a) and (c), $E_{l a b}=121 \mathrm{MeV}$ and in (b) and (d), $E_{l a b}=142 \mathrm{MeV}$

scission points in lighter systems. This suggests that the predictions of the saddlepoint model and an extended Hauser-Feshbach type scission should be similar in this case. Here, we compare the barriers and decay widths of these different formulations of intermediate mass fragment emission.

The transition state model of intermediate mass fragment emission approximates the emission width in terms of the density of states at the saddle point barrier using a thermal excitation energy obtained by subtracting the collective rotational energy in the sticking limit. It takes into account no additonal effects of the angular momenta of the fragments nor of their relative motion. To estimate the emission width of the scission model of intermediate mass fragment emission, we extend the expression above to take into account all effects of (classical) angular momentum as well as energy conservation. We write

$$
\begin{aligned}
2 \pi \Gamma\left(\varepsilon_{0}, \vec{J}_{0} ; Z_{1} A_{1}, Z_{2} A_{2}\right) \rho_{0}\left(\varepsilon_{0}, \vec{J}_{0}\right)= & \frac{1}{2(2 \pi \hbar)^{2}} \int \delta(\hat{r} \cdot \vec{L}) d^{3} L d \Omega d e_{r} \\
& \times \prod_{j=1}^{2}\left(\rho_{j}\left(\varepsilon_{j}, \overrightarrow{J_{j}}\right) d \varepsilon_{j} d^{3} J_{j}\right) \delta\left(\overrightarrow{J_{0}}-\vec{L}-\vec{J}_{1}-\vec{J}_{2}\right) \\
& \times \delta\left(\varepsilon_{0}-Q-e_{r}-\frac{L^{2}}{2 \mu R_{B}^{2}}-V_{B}-\varepsilon_{1}-\varepsilon_{2}\right) .
\end{aligned}
$$

To evaluate this expression, we use saddle point methods to evaluate most of the integrals, which also furnish the sticking limit of rotational motion at the saddle point, and then use the high energy limit of the Fermi gas level density to write the remaining product of level densities in terms of the saddle / scission level density that the GEMINI++ code expects. 
The statistical emission model taking into account classical angular momentum conservation was proposed by Ericson and Strutinsky. [13] The approximation to the partial width given above is equivalent to the assumption of a classical transmission coefficient, for which transmission above the barrier is unity and below the barrier is null. This should be reasonable at high excitation energies. The expression taking into account quantum barrier transmission has been used previously to describe intermediate mass fragment emission from light nuclei. [14]

\section{Results and discussion}

We use the GEMINI++ code to perform the calculations. This code was written in C++ by R. J. Charity, [10] and is the sucessor to his FORTRAN code GEMINI. The GEMINI++ code calculates the decay of the compound nucleus as a series of binary sequential emission of light or intermediate mass fragments or fission. The emission of intermediate mass fragments is in fact treated as asymmetric fission. The GEMINI++ code uses Monte Carlo sampling based on the partial decay widths of all processes. This has the advantage of allowing the inclusion of time differences between emissions, which permits the calculation of proximity effects between the fragments that can not be included in the conventional approach.

In 1 we compare GEMINI++ calculations of intermediate mass fragment charge and mass yields with the experimental data of [7,9] for the reaction ${ }^{32} \mathrm{~S}+{ }^{24} \mathrm{Mg}$ at $E_{c m}=51.6$ and $E_{c m}=60.5 \mathrm{MeV}$. The calculations shown by the blue circles were performed with the default parameters of the code,[10] using Sierk saddle-point barriers [11] and Moretto's transition density formalism[8]. The saddlepoint approximation to the fragment yields of Sanders et al. is given by the yellow points.[9] The pink triangles show the results from GEMINI++ with barriers obtained using the São Paulo potential at the scission radius. [12] The green diamonds represent the experimental data. We observe that the calculations tend to underestimate the intermediate mass fragment yields at the lower excitation energy of $1.25 \mathrm{MeV}$ per nucleon but are in better agreement at the higher energy of $1.79 \mathrm{MeV}$ per nucleon. Both values of the excitation energy are well below the range of about 3-4 MeV per nucleon above which multifragmentation becomes important.

The calculations using the GEMINI++ code do not present good agreement with the experimental data in the intermediate mass region at low values of the center-of-mass energy but improve in agreement as the energy increases. Part of the discrepancy is due to that fact that the excitation spectrum of the fragments is represented by a continuum density of states. No discrete states are included in the calculations. However, these states are extremely important at low energy, where liitle energy is available for exciting the fragments. In the calculations using the transition state density, the strong effect of the binding energy is taken into account by the adding a Wigner energy term to the the liquid drop energy. An alternative way to include this strong variation would be to incorporate shell effects in the level density.

The default mode of the code GEMINI++ uses the saddle point barriers of Sierk and Moretto's transition state density formalism[8] to predict the emission cross sections of complex fragments, which are appropriate for heavier systems. The transition-state model developed by Sanders et al. [9] is specifically adapted to the region of small mass and is quite successful in describing asymmetric fragmention in this region.

One of the basic differences between the saddle-point and scission-point calculations presented here are their barriers, which in both cases enter the density of states that determine the partial emission widths. As the deformation of a nucleus increases, it eventually reaches a form for which the surface tension can no longer counteract the Coulomb repulsion. This is the saddle point. For larger deformations, the nucleus will separate into two fragments, which occurs at the scission point. The 

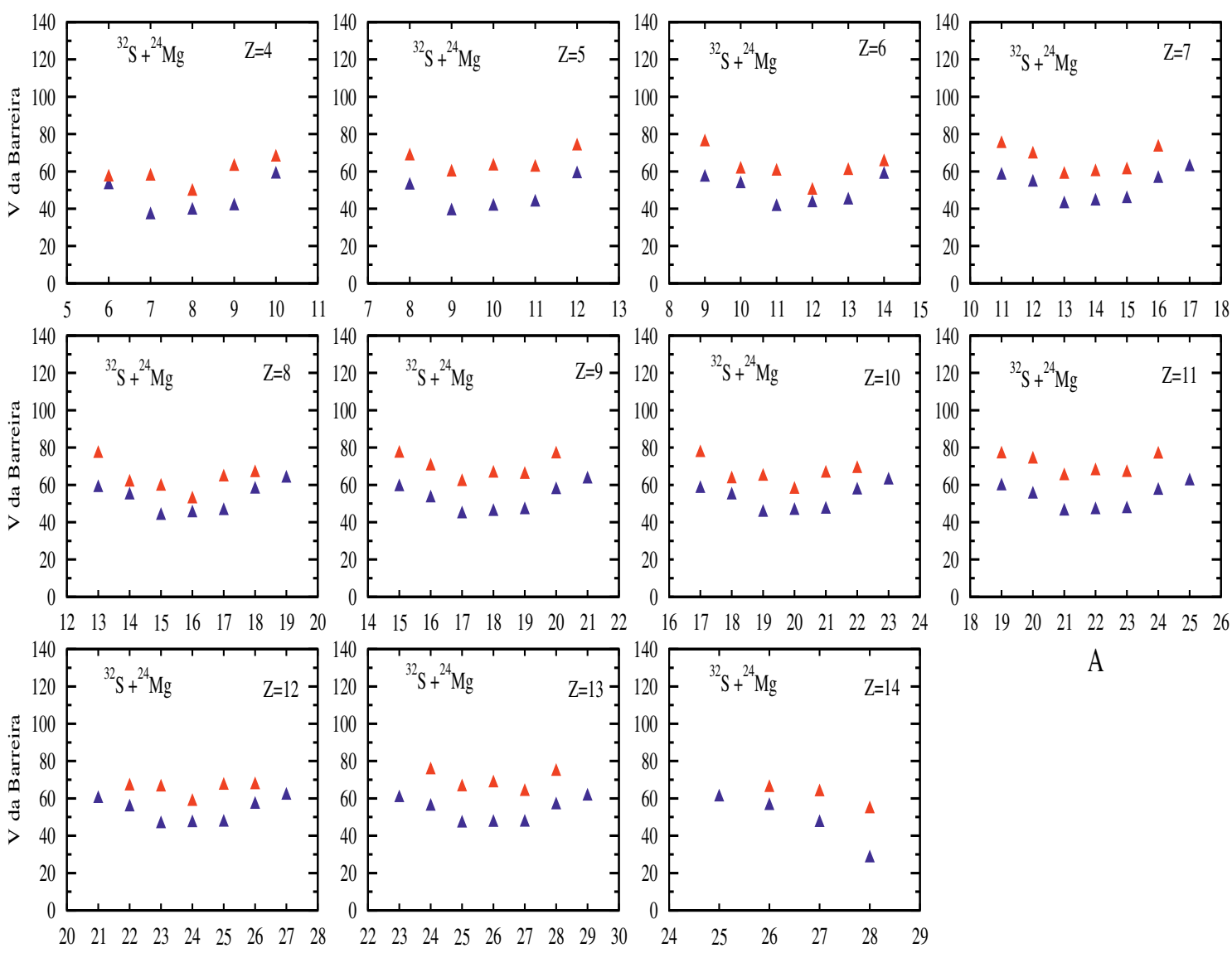

A

A
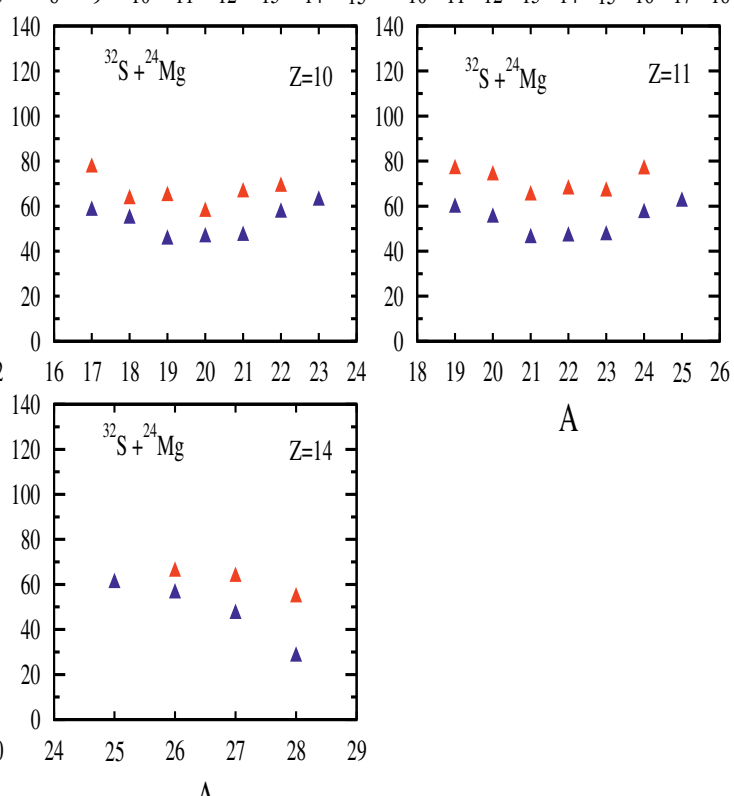

A

Figure 2. Comparison between the Sierk saddle-point barriers (blue triangles) and scission barriers of the São Paulo potential (red triangles) for the ${ }^{32} \mathrm{~S}+{ }^{24} \mathrm{Mg}$ reaction, as a function of the charge and mass of the fragment of smaller charge.

saddle point possesses a special stability to deformations in certain directions, for which the energy passes through a minimum.

For light nuclei, the deformations at the saddle and scission points are very similar. In these cases, the deformation necessary for instability is almost the same as that needed to split the nucleus in two fragments. As the mass and charge of the nucleus increases the difference in deformation at the two points increases as well. This is due to the fact that the Coulomb repulsion is of longe range, while the attractive surface tension is due to the short-range nuclear force. Thus the instability that leads to the fission of heavy nuclei occurs at a much smaller deformation than that at which the fragments actually separate. One would thus expect the saddle-point and scission-point barriers of light nuclei to be 
similar, while the saddle-point barrier of heavier nuclei should be much higher than the scission-point barrier.

In 2 we compare the Sierk saddle-point barriers (blue triangles) with the scission barriers of the São Paulo potential (red triangles) for the ${ }^{32} \mathrm{~S}+{ }^{24} \mathrm{Mg}$ reaction, as a function of the charge and mass of the fragment of smaller charge. Although the results for both barriers follow very similar trends as a function of the mass, we find the scission barriers of the São Paulo potential to be large than the saddle point barriers, rather than smaller, as would be expected. This is in part due to the fact that fragment deformation is included in the definition of the saddle point geometry, which is based on a full calculations of the energy, while the fragments of the scission calulation are assumed to be spherical. However, irregularities in the differences between the two barriers suggest that the saddlepoint barriers might still contain some contribution of the fragment energies.

In the future, we plan to extend the comparision of the barriers to heavier compound systems. We also plan to extend our calculations of intermediate mass fragment emission to higher excitation energies and to heavier compound nuclear systems and to compare the results with those of the statistical multifragmentation model as well as with experimental data. We also plan to improve our implementation of the scission-point intermediate mass fragment emission model by including fragment deformation and the effects of nuclear expansion at high temperatute in the determination of the scission point and intermediate mass fragment emission.

\section{References}

[1] J. P. Bondorf, R. Donangelo, I. N. Mishustin, C. J. Pethick, H. Schulz and, K. Sneppen, Nucl Phys. A443, 321 (1985).

[2] J. Bondorf, R. Donangelo, I. N. Mishustin, H. Schulz, Nucl.Phys. A444, 460 (1985).

[3] H. W. Barz, J. P. Bondorf, R. Donangelo, I. N. Mishustin, H. Schulz, Nucl.Phys. A448 753, (1986).

[4] A. S. Botvina, A. S. Iljinov, I. N. Mishustin, J. P. Bondorf, R. Donangelo, K. Sneppen, Nucl. Phys. A475, 663 (1987).

[5] B. V. Carlson, F. T. Dalmolin, M. Dutra, R. Donangelo, S. R. Souza, D. A. Toneli, CERN Proceedings 2012-002, 285 (2012).

[6] T. J. Santos, B. V. Carlson, AIP Conference Proceedings 1529, 284 (2013).

[7] T. Matsuse, C. Beck, R. Nouicer, D. Mahboub, Phys. Rev. C 55, 1380 (1997).

[8] L. G. Moretto, Nucl. Phys. A247, 211 (1975).

[9] S. J. Sanders, Phys. Rev. C 44, 2676 (1991).

[10] R. J. Charity, in Proceedings of the Joint ICTP-IAEA Advanced Workshop on Model Codes for Spallation Reactions (IAEA, Triste, Italy, 2008); edited by D. Filges, S. Leray, Y. Yariv, A. Mengoni, A. Stanculescu and G. Mank, INDC(NDS)-530, p. 139.

[11] A. J. Sierk, Phys. Rev. Lett. 55, 582 (1985).

[12] L. C. Chamon, B. V. Carlson, L. R. Gasques, D.Pereira, C. D. Conti, M. A. G. Alvarez, M. S. Hussein, Phys.Rev. C 66, 014610(2002).

[13] T. Ericson and V. Strutinsky, Nucl. Phys. 8, 284 (1958); 9, 689 (1958).

[14] B.V. Carlson, O. Civitarese, M. S. Hussein, A. Szanto de Toledo, Ann. Phys. (N.Y.) 169, 167 (1986). 